\title{
On the Atomic Kinetic Energies in Ceramic Oxides
}

\author{
Yacov Finkelstein ${ }^{*}$, Raymond Moreh ${ }^{2}$, Rony Shneck ${ }^{3}$ \\ ${ }^{1}$ Chemistry Division, Nuclear Research Center-Negev, Beer-Sheva, Israel \\ ${ }^{2}$ Department of Physics, Ben-Gurion University of the Negev, Beer-Sheva, Israel \\ ${ }^{3}$ Department of Materials and Engineering, Ben-Gurion University of the Negev, Beer-Sheva, Israel \\ Email: ^finklfam@gmail.com
}

How to cite this paper: Finkelstein, Y., Moreh, R. and Shneck, R. (2017) On the Atomic Kinetic Energies in Ceramic Oxides. Journal of Materials Science and Chemical Engineering, 5, 17-27. https://doi.org/10.4236/msce.2017.512002

Received: October 17, 2017

Accepted: December 5, 2017

Published: December 8, 2017

Copyright ( $) 2017$ by authors and Scientific Research Publishing Inc. This work is licensed under the Creative Commons Attribution International License (CC BY 4.0).

http://creativecommons.org/licenses/by/4.0/

\section{(c) (i) Open Access}

\begin{abstract}
We calculated the mean atomic kinetic energy, $\langle\operatorname{Ke}(\mathrm{X})\rangle$, of the $\mathrm{X}$ atom $(\mathrm{X}=$ $\mathrm{Si}, \mathrm{Ti}, \mathrm{Hf}, \mathrm{O}$ ) in some ceramic oxides, $\mathrm{SiO}_{2}, \mathrm{TiO}_{2}$ and $\mathrm{HfO}_{2}$ using the published partial vibrational density of states (PVDOS). These were simulated by means of lattice dynamics, molecular dynamics and density functional theory. The predicted values are compared to those recently obtained by electron Compton scattering (ECS), with an overall good agreement of $\sim 4 \%$. In accord with calculations, the ECS measurements reveal a small, but detectable, dependence of $\langle\operatorname{Ke}(\mathrm{X})>$ on the fine structural details of the oxide, e.g. whether it is in a crystalline or amorphous form, and whether it exhibits surface or bulk characteristics. This study illustrates the limitations and the potential of PVDOS simulations in predicting experimental atomic kinetic energies, and can be viewed as a promising approach for elucidating valuable structural and dynamical information of ceramic oxides and other materials.
\end{abstract}

\section{Keywords}

Ceramic Oxides, Atomic Kinetic Energy, Vibrational Density of States, Electron Compton Scattering

\section{Introduction}

Ceramic oxides such as $\mathrm{SiO}_{2}, \mathrm{TiO}_{2}$ and $\mathrm{HfO}_{2}$ are fundamental dielectrics amongst a large family of atomic layer deposition (ALD) materials commonly used in semiconductors and micro-/nano-electronics technologies. The complexity of these technologies demands in some cases, cross-disciplinary research studies. In the materials front, understanding the origin of their unique fundamental properties at atomic and molecular levels is important for the realization 
of the full potential of the fruition of eventual technological applications. Of key importance are the dynamical properties of ceramic oxides which may be studied via their vibrational spectra using IR, Raman and inelastic neutron scattering (INS) that provide fingerprints for clarifying their structure. In that respect, the vibrational density of states (VDOS), and in particular the partial VDOS (PVDOS) of specific atoms, are important to explore. An illustrative example is $\mathrm{HfO}_{2}$ which is utilized in resistive switching memories in thin film devices, exhibiting reversible metal-insulator transitions [1]. This switching phenomenon is not yet fully understood and is speculated to be governed by percolation of oxygen vacancies [2]. Understanding the hoping mechanism of the O-atoms between surface oxygen vacancies in $\mathrm{HfO}_{2}$ involves the study of hafnia PVDOS [3]. Another exemplifying case is that of amorphous silica $\left(\alpha-\mathrm{SiO}_{2}\right)$ which is widely used in semiconductor devices and optical fibers. Due to its disordered nature, the molecular motions taking place in $\alpha$ - $\mathrm{SiO}_{2}$, where surface librations are a key factor [4], have not yet been completely elucidated due to current experimental limitations. Nonetheless, some valuable structural information may be achieved through computational simulations of its vibrational spectra. Recently, calculations comprising VDOS and PVDOS, were used to study average atomic kinetic energies, $\langle\mathrm{KE}\rangle$, in some benchmark systems involving hydrogen bonds (HBs), e.g. in ice Ih and liquid water [5], highly compressed ice VII [6], ferroelectric crystals of the KDP-type [7], the $\mathrm{Rb}_{3} \mathrm{H}\left(\mathrm{SO}_{4}\right)_{2}$ superprotonic conductor [7] and water nano-confined in Beryl [8]. Very recently, the same idea was also applied for studying the anisotropy of $\mathrm{Ke}(\mathrm{H})$ in the ferro- and para-electric phases of $\mathrm{CDP}\left(\mathrm{CsH}_{2} \mathrm{PO}_{4}\right)$ [9]. A recent comprehensive review may be found in Ref. [10]. In fact, studies utilizing KE calculations go way back to the 90th utilizing nuclear resonance photon scattering NRPS [11] of ${ }^{15} \mathrm{~N}$ [12] and ${ }^{13} \mathrm{C}$ [13]. Interestingly it was applied to study molecular orientations of gases adsorbed on Grafoil ${ }^{\varpi}$ and Papyex $^{\oplus}$ [14] in various systems [15] [16] as well as in graphite intercalation compounds [17].

The calculations of $\langle\mathrm{KE}\rangle$ in these early studies relied on discrete characteristic frequencies of the system as measured using IR, Raman and inelastic n-scattering (INS). Only recently, with the advent of computational capabilities, a new approach was adopted, relying on the continuum of vibrational states (phonons) of the studied atom. The continuum, represented by the pVDOS of the atom is simulated by using DFT, molecular (MD) and lattice (LD) dynamics. Altogether, the validity of the above approach is strongly justified by the very good agreement obtained with photon-, neutron- and electron scattering techniques in many systems. In fact, ECS has been shown to be a powerful tool for testing KE calculations [18] [19] [20] [21] [22] including cases such as $\mathrm{TiO}_{2}$ [21] and $\mathrm{SiO}_{2}$ [34] ceramics. It was thus interesting to see to what extent this approach could be utilized, to distinguish between different ceramic forms of $\mathrm{TiO}_{2}$ and $\mathrm{SiO}_{2}$.

The ECS uses electrons with incident energies in the range 2 to $40 \mathrm{keV}$, and its capabilities were recently illustrated for low Z-elements using samples such as 
$\mathrm{H}_{2} \mathrm{O}$ [23], $\mathrm{NH}_{3}$ and $\mathrm{CH}_{4}$ [24]. The measured $<\mathrm{KE}>$ values in these molecular systems closely agreed with those predicted using a semi-empirical (SE) method. This later method assumes the harmonic approximation (HA) and a decoupling between the various modes contributing to $\langle\mathrm{KE}\rangle$, the input data are taken from experiment [10]. Note that the SE approach utilizing simulated PVDOS was found to correctly reproduce $\mathrm{Ke}(\mathrm{H})$ values even in strongly anharmonic systems such as in KDP type crystals [7], $\mathrm{Rb}_{3} \mathrm{H}\left(\mathrm{SO}_{4}\right)_{2}$ [7] and $\mathrm{H}_{2} \mathrm{O} @$ Beryl [8], indicating that anharmonicity has probably only small effect on the atomic kinetic energy [10].

In the present study we utilize the SE approach to deduce $\mathrm{Ke}(\mathrm{X})$ values of the atomic constituents in $\mathrm{SiO}_{2}, \mathrm{TiO}_{2}, \mathrm{HfO}_{2}$, by using their literature simulated PVDOS, and show that they are in good agreement with recent ECS measurements. An attempt is made to assign the measured values to specific structural characteristics of the studied oxides.

\section{Experimental Details}

Full experimental details of the ECS technique may be found elsewhere. [18] [19] [20] [21] [22] Here we just mention that the ECS measurements involved the use of electron beams with energies ( $E e=1$ to $40 \mathrm{keV}$ ) having low thermal spread and high resolution electron spectrometers. The mean kinetic energy of the scattering atom $\mathrm{Ke}(\mathrm{X})$ causes a Doppler broadening $\Delta \mathrm{e}$ of the scattered electrons; the two are related by $\Delta e=\sqrt{\frac{4}{3} \operatorname{Ke}(\mathrm{X}) \operatorname{Er}(\mathrm{X})}$, with $\operatorname{Er}(\mathrm{X})$ the recoil energy of the $\mathrm{X}$ atom caused by the scattered electron. In the following we explain how the atomic kinetic energies were calculated.

\section{Mean Atomic Kinetic Energy Calculations}

In a molecular solid the distribution of the vibrational states (phonons) is described by the VDOS in which each atom takes a fraction. Theoretically, the participation of each atom in the total VDOS is best described by projecting the simulated total VDOS on that atom, i.e. by the partial VDOS (PVDOS) hereby denoted by $g_{x}(v)$. The kinetic energy $\mathrm{Ke}(\mathrm{X})$ of the $\mathrm{X}$ atom in a molecular solid is contributed by the external (translational and rotational (libration) modes) and internal molecular vibrations of the building block molecule and may be expressed as: [25]

$$
K e(\mathrm{X})=\frac{3}{2} \int_{v_{0}}^{v_{f}} g_{x}(v) \alpha(v) \mathrm{d} v / \int_{v_{0}}^{v_{f}} g_{x}(v) \mathrm{d} v
$$

With $\alpha(v, T)=\frac{h v}{2}\left[\left(\mathrm{e}^{h v / k T}-1\right)^{-1}+\frac{1}{2}\right]$ the kinetic energy of a quantum harmonic oscillator of frequency $v$ with $v_{0}$ and $v_{f}$ the boundaries of $g_{x}(v)$, T the thermodynamic temperature and $\mathrm{k}$ the Boltzmann constant.

\section{Results and Discussion}

In the literature, various calculations of the total VDOS of $\mathrm{XO}_{2}$ type oxide were 
made. Only in few, the PVDOS of its atomic constituents is simulated, from which $\mathrm{Ke}(\mathrm{X})$ and $\mathrm{Ke}(\mathrm{O})$ were deduced. Figure 1 depicts representative PVDOS simulated for the present studies. Note that in silica some modes occur in the $1000-$ $1200 \mathrm{~cm}^{-1}$ region while those of titania and hafnia the modes terminate at about $900 \mathrm{~cm}^{-1}$. Another feature common to all PVDOS is that the $\mathrm{O}$-atom, being the lighter partner, systematically shares the major part of the high frequency phonon states. Along other phonon states the situation is less regular: In silica, the Si atom shares the major part of the mid phonon states and vice versa at lower energies. This situation is illustrated in Figure 2 which shows the ratios between the PVDOS of the heavier element and of oxygen in all three oxides. It may be noted that the heavier the element, the larger part it shares in the low phonon states while on going to higher phonon states the situation reverses. Here too, the silica behaves differently, where along the low frequency phonons, up to $\sim 400 \mathrm{~cm}^{-1}$, the $\mathrm{Si}$ and $\mathrm{O}$ atoms share nearly equal fractions of the kinetic energy. On crossing $\sim 400 \mathrm{~cm}^{-1}$ the kinetic energy fraction of Si steeply increases. This indicates that in the vibrations of $\mathrm{SiO}_{2}$, the $\mathrm{Si}$ atom moves against the two oxygens $\left(\mathrm{O}_{2}\right)$ which has a higher mass, a situation which differs from that of the other oxides.

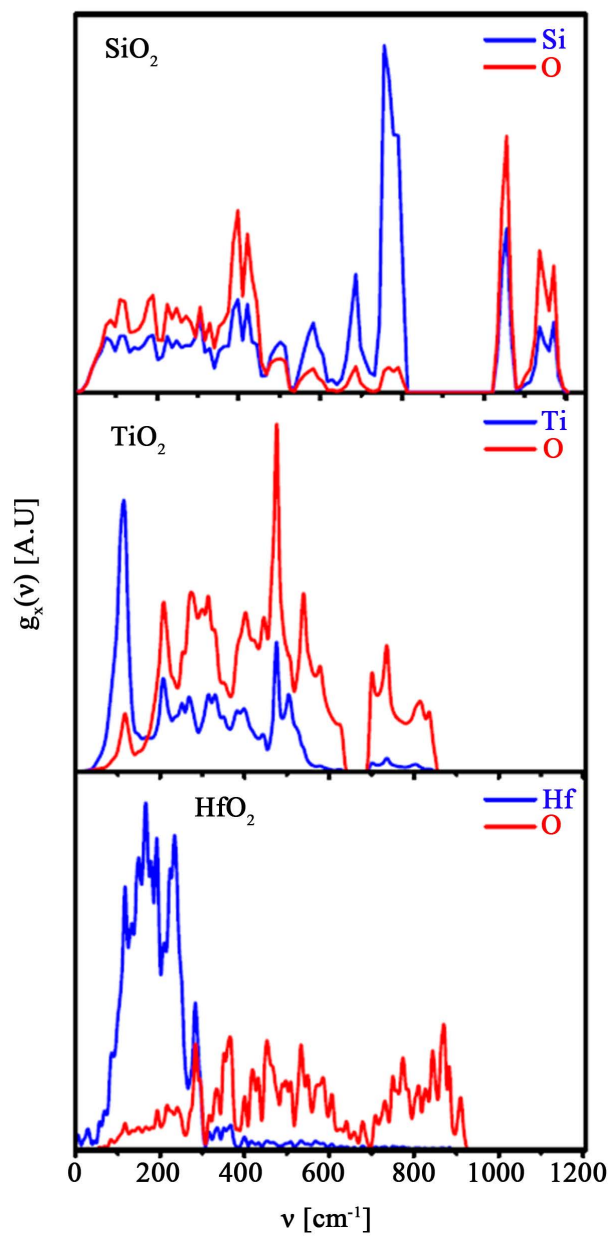

Figure 1. Simulated PVDOS of X (Si, Ti, Hf) and O atoms in $\alpha$-silica, [28] titania [29] (rutile) and hafnia [3]. 


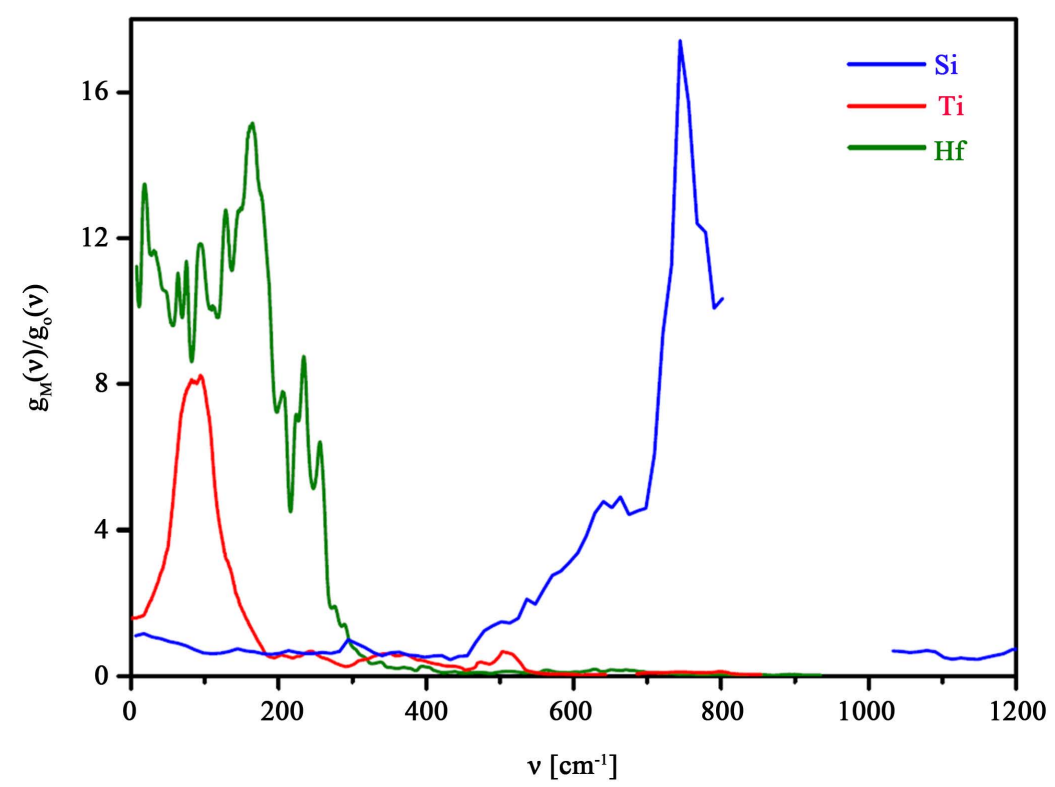

Figure 2. Ratio between the simulated VDOS of the $\mathrm{X}$ element $(\mathrm{X}=\mathrm{Si}, \mathrm{Ti}, \mathrm{Hf})$ and of the oxygen atom in $\alpha$-silica [28] (blue), titania (rutile) [29] (red) and hafnia [3] (green). The discontinuity between 800 and $1020 \mathrm{~cm}^{-1}$ in silica is due to the fact that no modes occur along this energy band (see Figure 1).

The simulated PVDOS of the various phases of $\mathrm{XO}_{2}(\mathrm{X}=\mathrm{Si}, \mathrm{Ti}, \mathrm{Ge}, \mathrm{Hf})$ were utilized for deducing $\operatorname{Ke}(\mathrm{X})$ and $\mathrm{Ke}(\mathrm{O})$ at room temperature using Equation (1). Table 1 summarizes the calculated values together with available ECS and NRPS experimental results. Note that all simulation techniques yield fairly close $\mathrm{KE}$ values for the $\mathrm{X}$-atoms.

The NRPS and ECS results of Table 1 lack the $\mathrm{Ke}(\mathrm{Ti})$ and $\mathrm{Ke}(\mathrm{Hf})$ values respectively. This is because the NRPS method is unique in the sense that it is a random nuclear resonance process and hence can only measure the KE of a single isotope of $\mathrm{Ti}$ (which happens to be ${ }^{48} \mathrm{Ti}$ ) in $\mathrm{TiO}_{2}$. In ECS on the other hand, electrons are scattered from all atomic constituents of the target. Hence, a reliable extraction of the mean kinetic energy of the Hf atoms becomes problematic due to lack of knowledge of the exact shape of the Hf elastic peak [35].

It is interesting to examine in Table 1 the values calculated by the same methodology, i.e. the DFT results of $\mathrm{SiO}_{2}$ and $\mathrm{TiO}_{2}$. These are shown to be very close to each other, indicating their reproducibility, despite the differences in the specific potentials and models used. An increase in these differences should however be expected at much lower temperatures, as the effect of the zero-point motion increases.

Altogether, Table 1 shows that the ECS results agree to less than 5\%, with predicted values. Note that $\operatorname{Ke}(\mathrm{X})$ and $\operatorname{Ke}(\mathrm{O})$ in molecular solids usually acquire intuitively expected values, i.e. Ke of the light partner is larger than the heavier one, as in ice where $\mathrm{Ke}(\mathrm{H}) \sim 152 \mathrm{meV}$ and $\mathrm{Ke}(\mathrm{O}) \sim 51 \mathrm{meV}$ [5] [36]. Note also that in the $\mathrm{HfO}_{2}$ result the isotopic composition of the oxygen in the sample was ${ }^{16} \mathrm{O}:{ }^{18} \mathrm{O}=1: 3$ (a $20 \mathrm{~nm} \mathrm{Hf}^{16} \mathrm{O}_{2}$ surface layer grown on a $60 \mathrm{~nm} \mathrm{Hf}^{18} \mathrm{O}_{2}$ layer). 
Table 1. Measured and calculated mean atomic kinetic energies $\langle\mathrm{Ke}(\mathrm{X})>$ of the $\mathrm{X}$ and $\mathrm{O}$ atoms in $\mathrm{XO}_{2}(\mathrm{X}=\mathrm{Ti}, \mathrm{Si}, \mathrm{Hf})$ at $300 \mathrm{~K}$. The $\mathrm{TiO}_{2}$ polymorphs include 4 high pressure phases (denoted by ${ }^{*}$ ). Explicit calculation techniques and phases are indicated. Note that the $\mathrm{Ke}(\mathrm{X})$ value for any atom at $\mathrm{T}=300 \mathrm{~K}$ of an ideal gas is $3 \mathrm{kT} / 2=38.6 \mathrm{meV}$ and that the excess kinetic energy is contributed by the zero-point motion which shows up in all oxides at $\mathrm{T}$ as high as $300 \mathrm{~K}$.

\begin{tabular}{|c|c|c|c|c|c|}
\hline \multirow{2}{*}{$\mathrm{X}$} & \multirow{2}{*}{ Technique } & \multirow{2}{*}{ Phase } & \multicolumn{3}{|c|}{$<\mathrm{Ke}(\mathrm{X})>[\mathrm{meV}]$} \\
\hline & & & $\mathrm{X}$ & $\mathrm{O}$ & Ref. \\
\hline \multirow{6}{*}{${ }^{28} \mathrm{Si}$} & MD & Amorphous Silica & 68.8 & 71.9 & {$[4]$} \\
\hline & DFT & Vitreous (fused) Silica (v-SiO $\left.{ }_{2}\right)$ & 67.3 & 64.0 & [26] \\
\hline & LD & $\alpha$-Cristobalite & 68.7 & 66.3 & [27] \\
\hline & DFT & $\alpha$-Quartz & 65.2 & 62.2 & [28] \\
\hline & & Average & 67.5 & 66.1 & \\
\hline & $E C S$ & Amorphous Silica & $68 \pm 2$ & $61 \pm 3$ & [21] \\
\hline \multirow{13}{*}{${ }^{48} \mathrm{Ti}$} & \multirow{7}{*}{ DFT } & Anastase & 47.4 & 54.6 & [29] \\
\hline & & $\mathrm{TiO}_{2} \mathrm{II}^{*}$ (Columbite) & 47.1 & 54.3 & [29] \\
\hline & & $\mathrm{MI}^{\star}($ Baddeleyite $)$ & 48.0 & 56.2 & [29] \\
\hline & & $\mathrm{OI}^{*}($ Orthorhombic I) & 48.1 & 57.1 & [29] \\
\hline & & $\mathrm{OII}^{*}($ Cotunnite $)$ & 47.6 & 57.0 & [29] \\
\hline & & & 46.3 & 53.9 & [29] \\
\hline & & Rutile & 47.3 & 56.2 & [30] \\
\hline & \multirow{4}{*}{ MD } & & 44.8 & 54.5 & [31] \\
\hline & & Amorphous Rutile & 48.7 & 58.1 & [32] \\
\hline & & Rutile Nanoparticle & 49.0 & 58.1 & {$[32]$} \\
\hline & & Average & 47.4 & 56.0 & \\
\hline & NRPS & Rutile & $44.7 \pm 2$ & & [33] \\
\hline & ECS & Polycrystalline Rutile & $48 \pm 2$ & $53 \pm 3$ & [34] \\
\hline \multirow{2}{*}{${ }^{178} \mathrm{Hf}$} & DFT & 1:7 (4-fold:3-fold) ${ }^{16} \mathrm{O}$ vacancies & 42.5 & 62.5 & [3] \\
\hline & ECS & 20/60 $\mathrm{nm} \mathrm{Hf}^{16} \mathrm{O}_{2} / \mathrm{Hf}^{18} \mathrm{O}_{2}$ surface bilayer & & $63 \pm 3$ & [35] \\
\hline
\end{tabular}

In cases where only the total VDOS is available, the use of Equation (1) yields the average kinetic energy $<\mathrm{KE}>$ per atom of the compound, which is valuable for testing the ECS measured values. By combining the measured INS low [37] and high [38] phonon frequencies of $\mathrm{v}^{-\mathrm{SiO}_{2}}$ at room temperature, $\langle\mathrm{KE}\rangle=63.9$ $\mathrm{meV}$ is deduced which agrees with the average measured ECS values of $\alpha$-Silica, being $63.3 \pm 2 \mathrm{meV}$. Following the same procedure for Ke deuced from the DFT of $\mathrm{v}-\mathrm{SiO}_{2},[26]$ yields $\langle\mathrm{KE}>=65.1 \mathrm{meV}$ which agrees with experiment to within $1.8 \%$ [37] [38].

Finally, it may be seen that $\operatorname{Ke}(\mathrm{X})$ in oxides systematically decreases with increasing mass of X. Note also that the OX bonds in oxides (Table 1) are strong covalent bonds and may be compared with Ke values of moderate ionic com- 
pounds (as in KDP type ferroelectrics and super-protonic conductors [7]) and in weak metallic bonds. An interesting $\operatorname{Ke}(\mathrm{X})$ behavior emerges as depicted in Figure 3 showing the average calculated values and the ECS results of Table 1 including those of $\mathrm{Li}$ and $\mathrm{Ca}$ (in carbonate forms) [21]. In addition, estimates of $\mathrm{Ke}(\mathrm{X})$ for pure metallic Debye solids are given in Figure 3 (see Ref. [7]) showing that Ke of covalently bonded metals in the present study are clearly higher than those in ionic and metallic solids. This observation strengthens the validity of our approach of deducing atomic KE values. It is interesting to see if the atomic kinetic energy is sensitive to structural differences. Ghuman et al. [32] used MD and $\mathrm{LD}$ to study the VDOS of $3 \mathrm{~nm}$ rutile and amorphous $\mathrm{TiO}_{2}$ nanoparticles. Representative results are given in Figure 4.

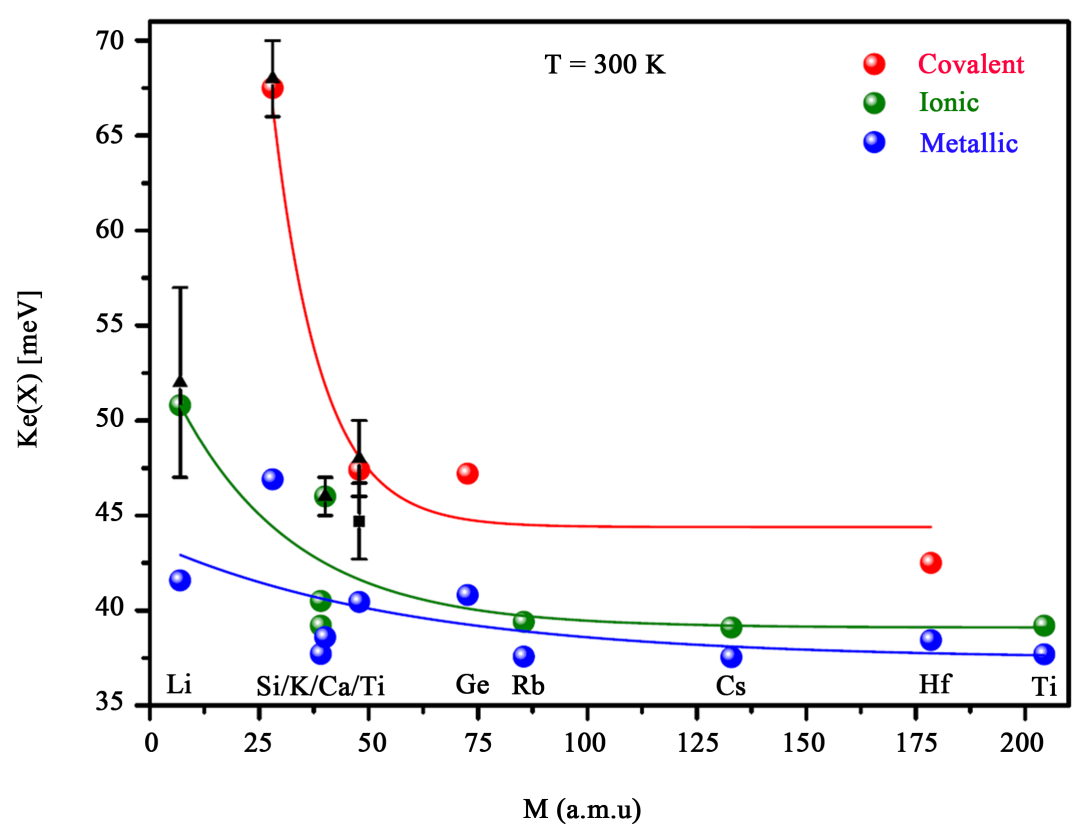

Figure 3. Calculated (full circles) and experimental (ECS-triangles, NRPS-Square) room temperature kinetic energies of the elements in the oxide samples (current study-red), in ferroelectrics and protonic superconductors (green [7] [21]) and in pure metals. [7] Solid curves are drawn to lead the eye.

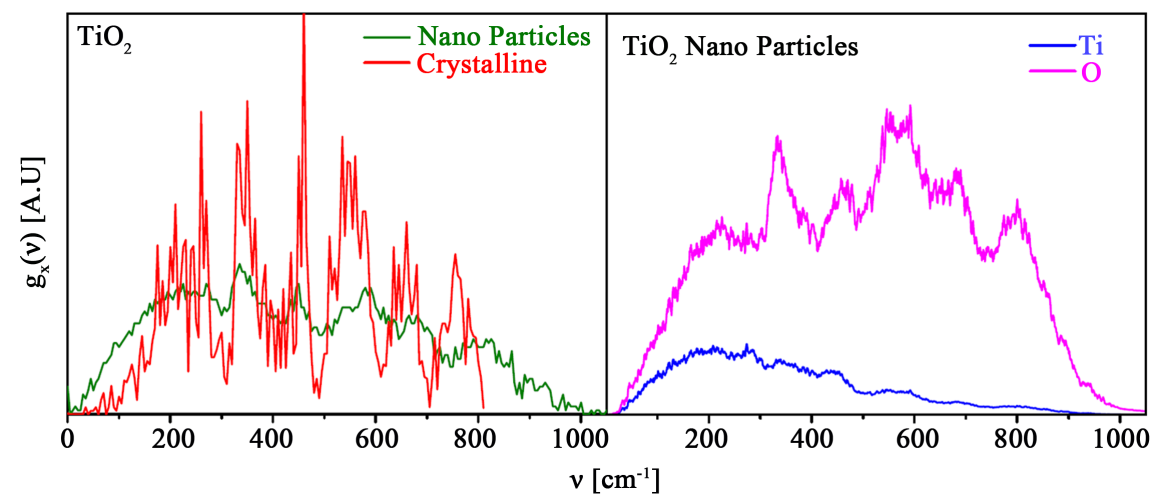

Figure 4. Left: Total VDOS of $3 \mathrm{~nm}$ rutile particles (green) and of rutile supercell (red). Right: PVDOS of the Ti (blue) and O (magenta) atoms in rutile nanoparticles. 
Figure 4 shows that nanoparticles feature a smeared blue shifted VDOS (by ca. $200 \mathrm{~cm}^{-1}$ ) compared to that of the crystalline form (the supercell calculation), which could indicate a stronger $\mathrm{Ti}-\mathrm{O}$ stretching in the nano-particles form. Moreover, the $\mathrm{O}$ atom shares the major part of the VDOS along the whole phonon states regime. By introducing the data of Figure 4 into Equation (1) we find (Table 1) that $\mathrm{Ke}(\mathrm{Ti})$ in the crystalline (rutile) form is $54.9 \mathrm{meV}$ (averaged over 3 independent DFT simulations), in good agreement with the ECS measurement $(53 \pm 3 \mathrm{meV})$. In the nanoparticle and amorphous forms however, the MD simulated $\mathrm{Ke}(\mathrm{H})$ is found to be the same, $58.1 \mathrm{meV}$. With fair statistical significance, such higher value may be understood as due to the extension of the VDOS towards more energetic phonons, as mentioned above. Contrary to titania, the case of Hafnia may poorly hint for an opposite behavior: as in titania, the total VDOS of the amorphous form was found to be smeared and blue shifted (by ca. $100 \mathrm{~cm}^{-1}$ ) compared to the crystalline form, [39]. However the deduced $<\mathrm{KEs}>$ (not shown in Table 1) in amorphous and crystalline forms are 50.9 and 51.6 $\mathrm{meV}$ respectively. The case of silica is less definitive (Table 1 ); while $\mathrm{Ke}(\mathrm{Si})$ in amorphous silica $(68.8 \mathrm{meV})$ is very close to the fused form $(67.3 \mathrm{meV})$, it is also practically the same as that of the crystalline polymorph $\alpha$-Cristobalite $(68.7$ $\mathrm{meV})$, and is higher than the other crystalline polymorph $\alpha$-Quartz $(65.2 \mathrm{meV})$.

\section{Summary}

We show that the study of atomic kinetic energies, KE, of ceramic oxides using ECS scattering is a potentially valuable tool for understanding their molecular/lattice vibrations and dynamical behavior. This may be achieved by utilizing various theoretical modeling platforms such as LD, MD and DFT for high quality simulations of the partial and total VDOS. These were used for predicting Ke and comparing with ECS and NCS measured values. The methodology presented here may be applied for example to ceramic oxide based solid electrolytes, e.g. $\mathrm{ZrO}_{2}$, for which it is of great interest to explore the dynamics of structural atoms, mainly of oxygen, near vacancies. Of interest is also the velocity or characteristic frequency of the ions near vacancies. Both may be important factors in enhancing the electrical current, nonetheless leaving the solid insulating for electrons. Calculating the $\operatorname{Ke}(\mathrm{O})$ in crystalline electrolytes and its dependence on the concentration of the alloying cations, can thus provide a valuable test for the above suggested mechanism. It is our hope that ECS will evolve and improve in accuracy to make it possible to elucidate valuable structural information of ceramic oxides and inorganic materials of scientific and technological interest.

\section{Acknowledgements}

We would like to thank Prof. Haibin Su from Nanyang University, Singapore, Dr. S. L. Shang of Materials Science, Pennsylvania state university, USA, Dr. Yuhua Duan of National energy technology, DOE, Pittsburgh, Pennsylvania, USA, Dr. C. Wang of Fukui University, Fukui, Japan, Dr. Luigi Giacomazzi of 
CNR-IOM Democritos, SISSA, Trieste, Italy, Dr. jörn Wehinger of Geneva University, Switzerland, and Dr. Xuhui Luo of Texas University, Austin, Texas, USA for providing the VDOS raw data of the various ceramic oxides.

\section{References}

[1] Zhang, H., Liu, L., Gao, B., Qiu, Y., Liu, X., Lu, J., Han, R., Kang, J. and Yu, B. (2011) Gd-Doping Effect on Performance of $\mathrm{HfO}_{2}$ Based Resistive Switching Memory Devices Using Implantation Approach. Applied Physics Letters, 98, 42103-42105. https://doi.org/10.1063/1.3543837

[2] Waser, R., Dittmann, R., Staikov, G. and Szot, K. (2009) Redox-Based Resistive Switching Memories-Nanoionic Mechanisms, Prospects, and Challenges. Advanced Materials, 21, 2632-2663. https://doi.org/10.1002/adma.200900375

[3] Wang, Z., Yu, H. and Su, H. (2013) The Transport Properties of Oxygen Vacancy-Related Polaron-Like Bound State in $\mathrm{HfO}_{\mathrm{x}}$. Scientific Reports, 3, 3246. https://doi.org/10.1038/srep03246

[4] Wang, C., Tamai, Y. and Kuzuu, N. (2003) A Molecular Dynamics Study on Vibration Spectra of $\alpha-\mathrm{SiO}_{2}$ Surface. Journal of Non-Crystalline Solids, 321, 204-209. https://doi.org/10.1016/S0022-3093(03)00233-3

[5] Finkelstein, Y. and Moreh, R. (2014) Temperature Dependence of the Proton Kinetic Energy in Water between 5 and 673 K. Chemical Physics, 431-432, 58-63. https://doi.org/10.1016/j.chemphys.2014.01.004

[6] Finkelstein, Y. and Moreh, R. (2013) Proton Dynamics in Ice VII at High Pressures. The Journal of Chemical Physics, 139, 44716. https://doi.org/10.1063/1.4816630

[7] Finkelstein, Y., Moreh, R., Shang, S.L., Shchur, Y., Wang, Y. and Liu, Z.K. (2016) On the Mean Kinetic Energy of the Proton in Strong Hydrogen Bonded Systems. The Journal of Chemical Physics, 144, 54302. https://doi.org/10.1063/1.4940730

[8] Finkelstein, Y., Moreh, R., Shang, S.L., Wang, Y. and Liu, Z.K. (2017) Quantum Behavior of Water Nano-Confined in Beryl. The Journal of Chemical Physics, 146, 124307. https://doi.org/10.1063/1.4978397

[9] Finkelstein, Y., Moreh, R. and Shchur, Y. (2017) Anisotropy of the Proton Kinetic Energy in $\mathrm{CsH}_{2} \mathrm{PO}_{4}$ and $\mathrm{KH}_{2} \mathrm{PO}_{4}$. Surface Science, 668, 112-116.

https://doi.org/10.1016/j.susc.2017.10.028

[10] Finkelstein, Y. and Moreh, R. (2017) Applying Semi-Empirical Quantum Harmonic Calculations for Studying the Atomic Kinetic Energies in Hydrogen Bonded Systems. Current Physical Chemistry, 7, 1.

[11] Moreh, R., Shahal, O. and Volterra, V. (1976) Effect of Molecular Binding on the Resonance Scattering of Photons from the $6.324 \mathrm{MeV}$ level in ${ }^{15} \mathrm{~N}$. Nuclear Physics $A$, 262, 221-230. https://doi.org/10.1016/0375-9474(76)90616-3

[12] Finkelstein, Y., Moreh, R., Nemirovsky, D., Shahal, O. and Tobias, H. (1999) Adsorption of $\mathrm{N}_{2}$ Monolayers on Papyex in the Liquid and Vapor Phases. Surface Science, 443, 89-98. https://doi.org/10.1016/S0039-6028(99)00990-5

[13] Moreh, R., Beck, O., Jäger, D., Finkelstein, Y., Kneissl, U., Margraf, J., Maser, H. and Pitz, H. (1997) Effective Temperature of Amorphous Carbon Studied Using Nuclear-Resonance Photon Scattering. Physical Review B, 56, 187-193. https://doi.org/10.1103/PhysRevB.56.187

[14] Finkelstein, Y., Moreh, R. and Shahal, O. (1999) Out-of-Plane Orientation of Multilayer $\mathrm{N}_{2}$ Films Adsorbed on Grafoil at 20 K. Surface Science, 437, 265-276. 
https://doi.org/10.1016/S0039-6028(99)00724-4

[15] Moreh, R., Pinto, H., Finkelstein, Y. and Beguin, F. (1996) Tilt of $\mathrm{N}_{2}$ Molecules Physintercalated into $\mathrm{C}_{24} \mathrm{~K}$ and $\mathrm{C}_{24} \mathrm{Rb}$. Journal of Physics and Chemistry of Solids, 57, 909-913. https://doi.org/10.1016/0022-3697(95)00372-X

[16] Finkelstein, Y. and Moreh, R. (1999) Anisotropic Nuclear-Resonance Photon Scattering from a Single Crystal of $\mathrm{NaNO}_{2}$. Physical Review B, 59, 6211. https://doi.org/10.1103/PhysRevB.59.6211

[17] Moreh, R., Finkelstein, Y. and Shechter, H. (1996) NO2 adsorption on Grafoil between 297 and 12 K. Physical Review B, 53, 16006-16012. https://doi.org/10.1103/PhysRevB.53.16006

[18] Moreh, R., Finkelstein, Y. and Vos, M. (2015) Electron Scattering as a Tool to Study Zero-Point Kinetic Energies of Atoms In Molecules. Nuclear Instruments and $\mathrm{Me}$ thods in Physics Research Section B: Beam Interactions with Materials and Atoms, 354, 37. https://doi.org/10.1016/j.nimb.2014.11.078

[19] Vos, M., Tökési, K. and Benkö, I. (2013) The Potential of Materials Analysis by Electron Rutherford Backscattering as Illustrated by a Case Study of Mouse Bones and Related Compounds. Microscopy and Microanalysis, 19, 576-586.

https://doi.org/10.1017/S143192761300041X

[20] Moreh, R., Finkelstein, Y. and Vos, M. (2015) Comparison between Electron and Neutron Compton Scattering Studies. EPJ Web of Conferences, 93, 02011.

https://doi.org/10.1051/epjconf/20159302011

[21] Vos, M., Marmitt, G.G., Finkelstein, Y. and Moreh, R. (2015) Determining the Band Gap and Mean Kinetic Energy of Atoms from Reflection Electron Energy Loss Spectra. The Journal of Chemical Physics, 143, Article ID: 104203. https://doi.org/10.1063/1.4929911

[22] Vos, M., Moreh, R. and Tokesi, K. (2011) The Use of Electron Scattering for Studying Atomic Momentum Distributions: The Case of Graphite and Diamond. The Journal of Chemical Physics, 135, Article ID: 24504. https://doi.org/10.1063/1.3607993

[23] Vos, M., Weigold, E. and Moreh, R. (2013) Elastic Electron Scattering from Water Vapor and Ice at High Momentum Transfer. The Journal of Chemical Physics, 138, Article ID: 44307. https://doi.org/10.1063/1.4775810

[24] Cooper, G., Hitchcock, A.P., Chatzidimitriou-Dreismann, C.A. and Vos, M. (2007) Electron Compton Scattering from Methane and Methane-d4. Journal of Electron Spectroscopy and Related Phenomena, 155, 28-34.

https://doi.org/10.1016/j.elspec.2006.11.001

[25] Finkelstein, Y., Beck, O., Moreh, R., Jäger, D., Kneissl, U., Margraf, J., Maser, H. and Pitz, H.H. (1998) Nuclear-Resonance-Photon-Scattering Study of the Effective Temperatures of Diamond and of Highly Oriented Pyrolytic Graphite. Physical Review $B, 58,4166-4172$. https://doi.org/10.1103/PhysRevB.58.4166

[26] Giacomazzi, L. (2007) First Principles Vibrational Spectra of Tetrahedrally-Bonded Glasses: $\mathrm{SiO}_{2}, \mathrm{GeO}_{2}$ and $\mathrm{GeSe}_{2}$. Universitàdegli Studi di Padova. http://infoscience.epfl.ch/record/98523

[27] Wehinger, B.B., Bosak, A.A., Refson, K., Mirone, A., Chumakov, A. and Krisch, M. (2015) Lattice Dynamics of $\alpha$-Cristobalite and the Boson Peak in Silica Glass. Journal of Physics. Condensed Matter, 27, Article ID: 305401. https://doi.org/10.1088/0953-8984/27/30/305401

[28] Duan, Y., Pfeiffer, H., Li, B., Romero-Ibarra, I.C., Sorescu, D.C., Luebke, D.R. and Halley, J.W. (2013) $\mathrm{CO}_{2}$ Capture Properties of Lithium Silicates with Different Ra- 
tios of $\mathrm{Li}_{2} \mathrm{O} / \mathrm{SiO}_{2}$ : An $\mathrm{Ab}$ Initio Thermodynamic and Experimental Approach. Physical Chemistry Chemical Physics, 15, 13538-13558. https://doi.org/10.1039/c3cp51659h

[29] Mei, Z.-G., Wang, Y., Shang, S.-L. and Liu, Z.-K. (2011) First-Principles Study of Lattice Dynamics and Thermodynamics of $\mathrm{TiO}_{2}$ Polymorphs. Inorganic Chemistry, 50, 6996-7003. https://doi.org/10.1021/ic200349p

[30] Sikora, R. (2005) Ab Initio Study of Phonons in the Rutile Structure of $\mathrm{TiO}_{2}$. Journal of Physics and Chemistry of Solids, 66, 1069-1073. https://doi.org/10.1016/j.jpcs.2005.01.007

[31] Lukačević, I., Gupta, S.K., Jha, P.K. and Kirin, D. (2012) Lattice Dynamics and Raman Spectrum of Rutile $\mathrm{TiO}_{2}$ : The Role of Soft Phonon Modes in Pressure Induced Phase Transition. Materials Chemistry and Physics, 137, 282-289. https://doi.org/10.1016/j.matchemphys.2012.09.022

[32] Ghuman, K.K., Goyal, N. and Prakash, S. (2013) Vibrational Density of States of TiO2 Nanoparticles. Journal of Non-Crystalline Solids, 373-374, 28-33. https://doi.org/10.1016/j.jnoncrysol.2013.04.022

[33] Jacob, I., Moreh, R., Shahal, O. and Wolf, A. (1987) Effective and Debye Temperatures of Ti in TiC, TiO2, and TiH2. Physical Review B, 35, 8-12. https://doi.org/10.1103/PhysRevB.35.8

[34] Vos, M., Marmitt, G.G. and Grande, P.L. (2016) A Comparison of ERBS Spectra of Compounds with Monte Carlo Simulations. Surface and Interface Analysis, 48, 415-421. https://doi.org/10.1002/sia.5948

[35] Vos, M., Grande, P.L., Venkatachalam, D.K., Nandi, S.K. and Elliman, R.G. (2014) Oxygen Self-Diffusion in HfO2 Studied by Electron Spectroscopy. Physical Review Letters, 112, Article ID: 175601. https://doi.org/10.1103/PhysRevLett.112.175901

[36] Finkelstein, Y. and Moreh, R. (2016) Proton Dynamics in Hydrogen Bonded Systems. Molecular Physics, 114, 2108-2114. https://doi.org/10.1080/00268976.2016.1184342

[37] Buchenau, U., Prager, M., Ncker, N., Dianoux, A.J., Ahmad, N. and Phillips, W.A. (1986) Low-Frequency Modes in Vitreous Silica. Physical Review B, 34, 5665-5673. https://doi.org/10.1103/PhysRevB.34.5665

[38] Carpenter, J.M. and Price, D.L. (1985) Correlated Motions in Glasses Studied by Coherent Inelastic Neutron Scattering. Physical Review Letters, 54, 441-443. https://doi.org/10.1103/PhysRevLett.54.441

[39] Luo, X., Zhou, W., Ushakov, S.V., Navrotsky, A. and Demkov, A.A. (2009) Monoclinic to Tetragonal Transformations in Hafnia and Zirconia: A Combined Calorimetric and Density Functional Study. Physical Review B, Condensed Matter and Materials Physics, 80. 